This item was submitted to Loughborough's Research Repository by the author.

Items in Figshare are protected by copyright, with all rights reserved, unless otherwise indicated.

\title{
Effect of user mobility and channel fading on the outage performance of UAV communications
}

PLEASE CITE THE PUBLISHED VERSION

https://doi.org/10.1109/LWC.2019.2955444

PUBLISHER

IEEE

VERSION

AM (Accepted Manuscript)

\section{PUBLISHER STATEMENT}

(c) 2019 IEEE. Personal use of this material is permitted. Permission from IEEE must be obtained for all other uses, in any current or future media, including reprinting/republishing this material for advertising or promotional purposes, creating new collective works, for resale or redistribution to servers or lists, or reuse of any copyrighted component of this work in other works.

\section{LICENCE}

\section{All Rights Reserved}

\section{REPOSITORY RECORD}

Khuwaja, Aziz Altaf, Yunfei Chen, and Gan Zheng. 2019. "Effect of User Mobility and Channel Fading on the Outage Performance of UAV Communications". Loughborough University.

https://hdl.handle.net/2134/13244549.v1. 


\title{
Effect of User Mobility and Channel Fading on the Outage Performance of UAV Communications
}

\author{
Aziz Altaf Khuwaja, Yunfei Chen, Senior Member, IEEE, Gan Zheng, Senior Member, IEEE
}

\begin{abstract}
Many wireless networks operate in a mobile environment with randomly moving user terminals. This letter analytically characterizes the impact of ground user mobility, propagation environment and channel fading on the outage performance of unmanned aerial vehicle (UAV) communications. Closed-form expressions for the outage probability using the random waypoint model for ground user mobility, UAV channel models for different propagation environments and the Nakagami$m$ model for fading channels are derived. Furthermore, the outage analysis takes into account the effect of co-channel interference by both the stationary and mobile users. Numerical results are presented to demonstrate the interplay between the communication performance and the system parameters.
\end{abstract}

Index Terms-Co-channel interference, Nakagami-m fading, outage probability, random waypoint model, UAV communications.

\section{INTRODUCTION}

In mobile networks, the presence of mobility can induce time-varying characteristics into the received signal [1]. The variation caused by the user mobility together with the fluctuation in the channel gain generated by multi-path fading can degrade the performance [2].

To capture the essential performance of unmanned aerial vehicle (UAV) communications, it is necessary to consider the effect of ground user mobility. Various models have been reported in the literature for user mobility in mobile and adhoc networks [3]. One such model is the random waypoint (RWP) model [4] that has been predominantly adopted in various communication systems. For example, reference [5] studied the impact of the receiver mobility on the signal-tonoise ratio (SNR) in an indoor scenario using visible light communications. For the physical layer security, reference [6] provided general expressions for the outage and capacity performances for a ground user that moves according to the RWP model. Another study in [7] analyzed the secrecy outage performance in the presence of moving interferers in a RWP network. For UAV communications, the authors in [8] derived statistics for the signal-to-interference ratio (SIR), in which multiple UAVs move according to the RWP model in the

The work of G. Zheng was supported in part by the Leverhulme Trust Research Project Grant under grant number RPG-2017-129.

A. A. Khuwaja is with the School of Engineering, University of Warwick, Coventry, CV4 7AL U.K. and also with the Department of Electrical Engineering, Sukkur IBA University, Sukkur 65200, Pakistan. (e-mail: A.khuwaja@warwick.ac.uk)

Y. Chen is with the School of Engineering, University of Warwick, Coventry, CV4 7AL U.K. (e-mail: Yunfei.Chen@warwick.ac.uk)

G. Zheng is with the Wolfson School of Mechanical, Electrical, and Manufacturing Engineering, Loughborough University, Loughborough, LE11 3TU U.K. (e-mail: g.zheng@lboro.ac.uk) vertical direction. However, none of these works has provided a comprehensive analysis on the effect of ground user mobility in the UAV communications.

Motivated by the above observations, this letter presents an analytical framework to investigate how ground user mobility, propagation environments, and fading channels affect the UAV communications in noise-only as well as interference-limited scenarios. Specifically, we derive closed-form expressions for the cumulative distribution function (CDF) and the outage probability of the UAV system. In this letter, the user mobility follows the RWP model, the large-scale fading follows the line-of-sight (LOS) and probabilistic LOS [9] propagation models, and the small-scale channel fading follows the Nakagami- $m$ model. The Gamma distribution is used to approximate the aggregate interference. Numerical results are presented to show how the performance changes with the system parameters.

\section{SySTEM MODEL}

Fig. 1 depicts the uplink of a UAV communication system that consists of an aerial base station positioned at an altitude of $h$ meters covering multiple ground users in a small cell. In order to save battery and to simplify the system, we consider the use of a static multi-rotor $\mathrm{UAV}^{1}$. For a two-dimensional (2D) Cartesian coordinate system, we assume that $O_{c}$ is the projection coordinate of the UAV at the center of the circular cell. During the communication with UAV, the desired and interfering ground users may be randomly moving inside the circular region, such that their spatial positions are determined by the distances $r_{U}$ and $r_{I}$ from the center within a range of $0 \leq r_{U} \leq D_{U}$ and $0 \leq r_{I} \leq D_{I}$, respectively. In this case, $r_{U}$ and $r_{I}$ are random variables, and $D_{U}$ and $D_{I}$ are their respective maximum radial distance.

\section{A. Channel Model}

This letter takes into account both the LOS and the probabilistic LOS channel models commonly used for UAV communications [11]. First, we assume that the ground-to-air (GA) channel is dominated by LOS conditions. Therefore, the received power at the UAV can be given as

$$
P_{U}^{A}=\frac{P_{t} \delta|g|^{2}}{d_{U}^{\alpha}}=\frac{P_{t} \delta|g|^{2}}{\sqrt{r_{U}^{2}+h^{2}}}
$$

\footnotetext{
[10].

${ }^{1}$ In some cases, UAV consume less energy in mobility than in hovering
} 


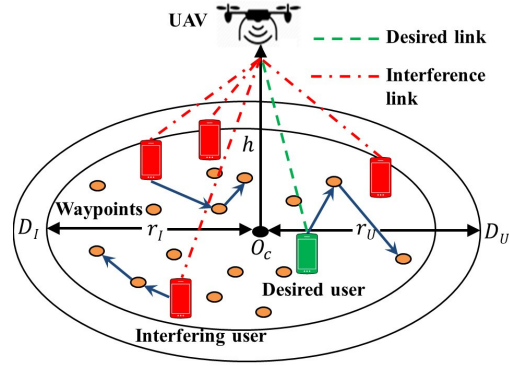

Fig. 1. System model.

where $d_{U}$ denotes the GA propagation distance between the ground user and UAV, $\delta=\left(\frac{c}{4 \pi f}\right)^{2}$ represents the frequencydependent channel power at the reference distance of 1 meter, $f$ is the carrier frequency, $c$ is the speed of light, $|g|^{2}$ is the fading power, $P_{t}$ is the ground user's transmission power, and $\alpha$ is the path-loss exponent which usually satisfies $2 \leq \alpha \leq 4$ between free space and obstructed propagation environments [12]. Thus, $\alpha=2$ and $\alpha=4$ can be considered as upper and lower limits of the performance, respectively. This model is useful in applications, where it is non-trivial to identify the environment (urban, dense-urban, etc.), for instance, in post-disaster areas with partial or complete infrastructural destruction [13]. Second, we use the probabilistic LOS model where the GA propagation is dependent on the environmental parameters and the elevation angle between the ground user and UAV. In this case, the received power at the UAV can be expressed as

$$
P_{U}^{B}=\frac{P_{t}|g|^{2}}{Q_{U}},
$$

where $Q_{U}=10^{\frac{P L_{U}}{10}}$ is the absolute power loss and $P L_{U}$ is the GA propagation path-loss given in [9] as

$$
P L_{U}=\frac{\left(\eta_{\mathrm{LOS}}-\eta_{\mathrm{NLOS}}\right)}{1+a_{1} e^{-b_{1}\left(\theta_{U}-a_{1}\right)}}+\xi_{U}
$$

and $\theta_{U}=\arctan \left(\frac{h}{r_{U}}\right)$ is the elevation angle, $\xi_{U}=$ $20 \log _{10}\left(\frac{4 \pi f}{c}\right)+20 \log _{10}\left(\sqrt{r_{U}^{2}+h^{2}}\right)+\eta_{\mathrm{NLOS}}, a_{1}, b_{1}, \eta_{\mathrm{LOS}}$, and $\eta_{\mathrm{NLOS}}$ are the constants related to the propagation environments. However, (3) is too complicated to analytically characterize the effect of the ground user mobility. Thus, we use the curve fitting approach to fit the probabilistic LOS channel for simplification. To this end, the curve fitting method yields a three-variable power function for $Q_{U}$ as

$$
Q_{U}=a_{2} r_{U}^{b_{2}}+c_{2},
$$

where $a_{2}, b_{2}$, and $c_{2}$ are the curve fitting parameters related to the environment and $\theta_{U}$.

To model the fading channel, we use the Nakagami- $m$ model due to its analytical tractability. This model is also reported in several measurement campaigns [11]. In our analysis, we consider normalized average fading power. Therefore, the probability density function (PDF) of the channel power follows a Gamma distribution as

$$
f_{|g|^{2}}(x)=\frac{m^{m} x^{m-1}}{\Gamma(m)} e^{-m x}, \quad x \geq 0
$$

where $m$ is the Nakagami- $m$ parameter which is assumed an integer in our work for simplicity and $\Gamma($.$) is the Gamma$ function.

\section{B. Mobility Model}

As shown in Fig. 1, we assume that user mobility follows the RWP model. As a result, the PDF of the desired user and interfering user distance is given in a polynomial form in [1, Table 1] and is summarized in [2] in an equivalent form of

$$
f_{r_{U}}\left(r_{U}\right)=\sum_{i=1}^{n} \beta_{i} \frac{r_{U}^{\alpha_{i}}}{D_{U}^{\alpha_{i}+1}}, \quad 0 \leq r_{U} \leq D_{U}
$$

and

$$
f_{r_{I}}\left(r_{I}\right)=\sum_{i=1}^{n} \beta_{i} \frac{r_{I}^{\alpha_{i}}}{D_{I}^{\alpha_{i}+1}}, \quad 0 \leq r_{I} \leq D_{I},
$$

respectively, where $n$ is determined by the dimension of space (1D, 2D, and 3D) considered, $\beta_{i}$ and $\alpha_{i}$ are constants determined by the user mobility in specific dimension. We assume all ground users are on a 2D surface, as shown in Fig. 1. Thus, $n=3$ with $\beta_{i}=\left[\frac{324}{73}, \frac{-420}{73}, \frac{96}{73}\right]$, and $\alpha_{i}=[1,3,5]$ [1]. It can be verified that integrals of (6) and (7) give 1.

\section{SNR ANALYSIS}

The received SNR at the UAV is given as $\lambda_{r}^{A}=\frac{E_{U}^{A}|g|^{2}}{\sqrt{1+\frac{r_{U}^{2}}{h^{2}}}}$ for LOS model by using (1) and $\lambda_{r}^{B}=\frac{E_{U}^{B}|g|^{2}}{\left(1+\frac{a_{2}}{c_{2}} r_{U}^{b_{2}}\right)}$ for probabilistic LOS model by using (2) and (4), where $E_{U}^{A}=\frac{P_{t} \delta}{N_{0}} h^{-\alpha}, E_{U}^{B}=$ $\frac{P_{t}}{N_{0} c_{2}}$, and $N_{0}$ is the noise power.

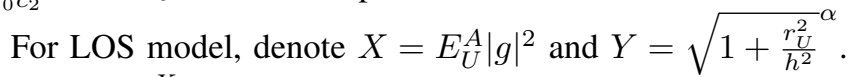
Let $Z=\frac{X}{Y}$, where $X$ and $Y$ are independent random variables. Hence, the $\mathrm{CDF}$ of $Z$ is given by

$$
F_{Z}(z)=\int_{1}^{{\sqrt{1+\left(D_{U} / h\right)^{2}}}^{\alpha}} \int_{0}^{z y} f_{X}(x) f_{Y}(y) d x d y .
$$

Substituting $\alpha=2$ in (8), the CDF of $Z$ can be derived using binomial expansion $(1+q)^{m}$ [14, eq.(1.110)] and the lower incomplete Gamma function $\gamma(.,$.$) [14, eq.(3.381.1)], as$

$$
\begin{aligned}
& F_{Z}(z)=\sum_{i=1}^{n} \frac{\beta_{i}}{\alpha_{i}+1}-\frac{e^{-\frac{z}{E_{U}^{A} / m}}}{2} \sum_{i=1}^{n} \sum_{j=0}^{m-1} \sum_{k=0}^{j} \frac{\beta_{i}}{\left(D_{U} / h\right)^{\alpha_{i}+1}} \times \\
& \frac{\left(\begin{array}{l}
k \\
j
\end{array}\right)}{j !} \frac{\gamma\left(\frac{\alpha_{i}+1}{2}+k, \frac{\left(D_{U} / h\right)^{2}}{E_{U}^{A} / m} z\right)}{\left(\frac{z}{E_{U}^{A} / m}\right)^{k+\frac{\alpha_{i}+1}{2}-j}} .
\end{aligned}
$$

Substituting $\alpha=4$ in (8), the CDF can be derived as

$$
F_{Z}(z)=\sum_{i=1}^{n} \frac{\beta_{i}}{\alpha_{i}+1}-\sum_{i=1}^{n} \sum_{s=1}^{4} \frac{\left(\frac{z}{E_{U}^{A} / m}\right)^{m-1}}{4\left(D_{U} / h\right)^{\alpha_{i}+1}} S_{s},
$$

where $G(\mu, \rho, \psi)=\frac{\gamma\left(m+\mu, \frac{\chi^{\rho}}{E_{U}^{A / m}} z\right)-\gamma\left(m+\mu, \frac{\chi^{\psi}}{E_{U}^{A} / m} z\right)}{\left(\frac{z}{E_{U}^{A} / m}\right)^{m+\mu}}, \chi=$ $\frac{D^{2}+h^{2}}{h^{2}}, S_{1}=G\left(-\frac{1}{2}, 2,0\right), S_{2}=2 \times G(1,0,2), S_{3}=$ $G\left(\frac{1}{2}, 2,0\right)$, and $S_{4}=G(0,0,2)$. 
For the probabilistic LOS model, let $X=E_{U}^{B}|g|^{2}, Y=$ $1+\frac{a_{2}}{c_{2}} r_{U}^{b_{2}}$, and $Z=\frac{X}{Y}$. The CDF of $Z$ can be derived as

$$
\begin{aligned}
& F_{Z}(z)=\sum_{i=1}^{n} \frac{\beta_{i}}{\alpha_{i}+1}-\frac{e^{-\frac{z}{E_{U}^{B} / m}}}{b_{2}} \sum_{i=1}^{n} \sum_{j=0}^{m-1} \sum_{k=0}^{j} \frac{\beta_{i}}{D_{U}^{\alpha_{i}+1}} \times \\
& \frac{\left(a_{2} / c_{2}\right)^{-\frac{\alpha_{i}+1}{b_{2}}}\left(\begin{array}{c}
k \\
j
\end{array}\right)}{j !} \frac{\gamma\left(\frac{\alpha_{i}+1}{b_{2}}+k, \frac{\left(a_{2} / c_{2}\right) D_{U}^{b_{2}}}{E_{U}^{B} / m} z\right)}{\left(\frac{z}{E_{U}^{B} / m}\right)^{k+\frac{\alpha_{i}+1}{b_{2}}-j}} .
\end{aligned}
$$

\section{SIR ANALYSIS}

Here, we consider the scenario when the desired ground user is surrounded by $L$ Nakagami- $m$ interfering users in two cases. In the first case, we assume independent and identically distributed static interfering users at approximately the same distance to the desired mobile user. In the second case, we consider the mobile interfering users moving randomly according to the RWP model. In both cases, the co-channel interference is incurred when the interfering users send signals to degrade the received signal at the UAV in the uplink. Thus, the aggregate interference ${ }^{2}$ at the UAV for LOS and probabilistic LOS channels is given as $V^{A}=\sum_{l=1}^{L} \frac{E_{I}^{A}\left|g_{l}\right|^{2}}{\sqrt{1+\frac{r_{I}^{2}}{h^{2}}}}$ and $V^{B}=\sum_{l=1}^{L} \frac{E_{I}^{B}\left|g_{l}\right|^{2}}{\left(1+\frac{a_{2}}{c_{2}} r_{I_{l}}^{b_{2}}\right)}$, respectively, where $E_{I}^{A}=$ $P_{t} \delta h^{-\alpha}$ and $E_{I}^{B}=\frac{P_{t}}{c_{2}}$. The aggregate interference can be well approximated by a Gamma random variable [2], [7]. Therefore, PDF of a Gamma distribution can be given as

$$
f_{V}(v)=\frac{v^{\vartheta-1}}{\Gamma(\vartheta) \gamma^{\vartheta}} e^{-\frac{v}{\gamma}}, \quad v \geq 0,
$$

where $\vartheta$ and $\gamma$ are the shape and scale parameters, respectively. For static interferers, $\vartheta=m L, \gamma=\frac{P_{t} \delta}{m{\sqrt{D_{I}^{2}+h^{2}}}^{\alpha}}$ for LOS model, and $\gamma=\frac{P_{t}}{m\left(a_{2} D_{I}^{b_{2}}+c_{2}\right)}$ for probabilistic LOS model. For mobile interferers, these parameters can be determined by moment-matching. Using (7) and [14, eq. (3.194.1)], in terms of the Gauss hypergeometric function ${ }_{2} F_{1}(\cdot, \cdot ; \cdot ; \cdot), \quad M_{A}=E\left\{\frac{1}{\sqrt{1+\left(r_{I} / h\right)^{2}}}\right\}=$ $\sum_{i=1}^{n} \frac{\beta_{i}}{\alpha_{i}+1}{ }_{2} F_{1}\left(\frac{\alpha}{2}, \frac{\alpha_{i}+1}{\alpha} ; \frac{\alpha_{i}+1}{\alpha}+1 ;-\left(D_{I} / h\right)^{2}\right), M_{B}=$ $E\left\{\frac{1}{1+\left(a_{2} / c_{2}\right) r_{I}^{b_{2}}}\right\}=\sum_{i=1}^{n} \frac{\beta_{i}}{\alpha_{i}+1}{ }_{2} F_{1}\left(1, \frac{\alpha_{i}+1}{b_{2}} ; \frac{\alpha_{i}+1}{b_{2}}+\right.$ $\left.1 ;-\left(a_{2} / c_{2}\right) D_{I}^{b_{2}}\right)$. Similarly, $N_{A}=E\left\{\frac{1}{\left(\sqrt{1+\left(r_{I} / h\right)^{2}}\right)^{2}}\right\}=$ $\sum_{i=1}^{n} \frac{\beta_{i}}{\alpha_{i}+1}{ }_{1} F_{1}\left(\alpha, \frac{\alpha_{i}+1}{\alpha} ; \frac{\alpha_{i}+1}{\alpha}+1 ;-\left(D_{I} / h\right)^{2}\right), \quad N_{B}=$ $E\left\{\frac{1}{\left(1+\left(a_{2} / c_{2}\right) r_{I}^{b_{2}}\right)^{2}}\right\}=\sum_{i=1}^{n} \frac{\beta_{i}}{\alpha_{i}+1}{ }_{2} F_{1}\left(2, \frac{\alpha_{i}+1}{b_{2}} ; \frac{\alpha_{i}+1}{b_{2}}+\right.$ $\left.1 ;-\left(a_{2} / c_{2}\right) D_{I}^{b_{2}}\right) . \quad E\left\{|g|^{2}\right\}=1, \quad$ and using [14, eq.(3.381.4)], $E\left\{|g|^{4}\right\}=1+\frac{1}{m}$. Thus, for LOS model, one has $E\{V\}=L E_{I}^{A} M_{A}$ and $E\left\{V^{2}\right\}=$ $L\left(E_{I}^{A}\right)^{2}(L-1) M_{A}^{2}+L\left(E_{I}^{A}\right)^{2}\left(1+\frac{1}{m}\right) N_{A}$. Finally, since $E\{V\}=\vartheta \gamma$ and $E\left\{V^{2}\right\}={ }^{m} \gamma^{2}-\vartheta^{2} \gamma^{2}$ from moment-matching, $\vartheta=\frac{L M_{A}^{2}}{\left(1+\frac{1}{m}\right) N_{A}-M_{A}^{2}}$ and

\footnotetext{
${ }^{2}$ For a tractable analysis, we assumes that the interference power is dominant as compared to noise [15].
}

$\gamma=E_{I}^{A}\left[\frac{N_{A}}{M_{A}}\left(1+\frac{1}{m}\right)-M_{A}\right]$. Similarly, for probabilistic LOS model, $\vartheta=\frac{L M_{B}^{2}}{\left(1+\frac{1}{m}\right) N_{B}-M_{B}^{2}}$ and $\gamma=E_{I}^{B}\left[\frac{N_{B}}{M_{B}}\left(1+\frac{1}{m}\right)-M_{B}\right]$.

Next, for LOS model, denote $Z_{I}=\frac{P_{U}^{A}}{V^{A}}$ as the received SIR. For $\alpha=2$, the $\mathrm{CDF}$ of $P_{U}^{A}$ i.e., $F_{P_{U}^{A}}\left(z_{I}\right)$ can be determined by replacing $E_{U}^{A}$ with $E_{I}^{A}$ in (9). Also, the PDF of $V_{A}$ can be obtained from (12). Since $P_{U}^{A}$ and $V_{A}$ are independent, the $\mathrm{CDF}$ of $Z_{I}$ is $F_{Z_{I}}\left(z_{I}\right)=\int_{0}^{\infty} F_{P_{U}^{A}}\left(v z_{I}\right) f_{V}(v) d v$. Thus, using [14, eq.(6.381.4)] and [14, eq.(6.455.2)], the CDF of $Z_{I}$ can be derived as

$$
\begin{aligned}
& F_{Z_{I}}\left(z_{I}\right)=\sum_{i=1}^{n} \frac{\beta_{i}}{\alpha_{i}+1}-\frac{1}{\Gamma(\vartheta) \gamma^{\vartheta}} \sum_{i=1}^{n} \sum_{j=0}^{m-1} \sum_{k=0}^{j} \frac{\beta_{i}\left(\begin{array}{c}
k \\
j
\end{array}\right)}{j !} \times \\
& \frac{\left(E_{I}^{A} / m\right)^{\vartheta} z_{I}^{j}\left(D_{U} / h\right)^{2 k} \Gamma(\vartheta+j)}{\left(\alpha_{i}+1+2 k\right)\left[\left(1+\left(D_{U} / h\right)^{2}\right) z_{I}+\frac{E_{I}^{A}}{m \gamma}\right]^{\vartheta+j}}{ }_{2} F_{1}(1, \vartheta+j ; \\
& \left.\frac{\alpha_{i}+1}{2}+k+1 ; \frac{z_{I}\left(D_{U} / h\right)^{2}}{\left(1+\left(D_{U} / h\right)^{2}\right) z_{I}+\frac{E_{I}^{A}}{m \gamma}}\right) .
\end{aligned}
$$

Similarly, for probabilistic LOS model, denote $Z_{I}=\frac{P_{U}^{B}}{V^{B}}$ as the received SIR, the CDF of $P_{U}^{B}$ i.e., $F_{P_{U}^{B}}\left(z_{I}\right)$ can be determined by replacing $E_{U}^{B}$ with $E_{I}^{B}$ in (11). Thus, the CDF of $Z_{I}$ can be derived as

$$
\begin{aligned}
& F_{Z_{I}}\left(z_{I}\right)=\sum_{i=1}^{n} \frac{\beta_{i}}{\alpha_{i}+1}-\frac{1}{\Gamma(\vartheta) \gamma^{\vartheta}} \sum_{i=1}^{n} \sum_{j=0}^{m-1} \sum_{k=0}^{j} \frac{\beta_{i}\left(\begin{array}{c}
k \\
j
\end{array}\right)}{j !} \times \\
& \frac{\left(E_{I}^{B} / m\right)^{\vartheta} z_{I}^{j}\left(\left(a_{2} / c_{2}\right) D_{U}^{b_{2}}\right)^{k} \Gamma(\vartheta+j)}{\left(\alpha_{i}+1+k b_{2}\right)\left[\left(1+\left(a_{2} / c_{2}\right) D_{U}^{b_{2}}\right) z_{I}+\frac{E_{I}^{B}}{m \gamma}\right]^{\vartheta+j}}{ }^{b_{2}} F_{1}(1, \\
& \left.\vartheta+j ; \frac{\alpha_{i}+1}{b_{2}}+k+1 ; \frac{z_{I}\left(a_{2} / c_{2}\right) D_{U}^{b_{2}}}{\left(1+\left(a_{2} / c_{2}\right) D_{U}^{b_{2}}\right) z_{I}+\frac{E_{I}^{B}}{m \gamma}}\right) .
\end{aligned}
$$

\section{Outage Performance}

Outage performance is defined in terms of outage probability when the received SNR or SIR falls below a predefined threshold $\lambda$ as

$$
\begin{gathered}
P_{\text {out }}=\operatorname{Pr}(Z<\lambda)=F_{Z}(\lambda) . \\
P_{\text {out }}=\operatorname{Pr}\left(Z_{I}<\lambda\right)=F_{Z_{I}}(\lambda) .
\end{gathered}
$$

where the CDF of $F_{Z}(z)$ and $F_{Z_{I}}\left(z_{I}\right)$ are given in Section III and Section IV, respectively.

\section{NumericAl RESUlts AND Discussion}

In this section, we analyze the performance for different channel conditions and mobility. As an illustrative example, let $h=100$ meters, $P_{t}=30 \mathrm{dBm}$, and $N_{0}=-57 \mathrm{dBm}$. Also, for $f=2 \mathrm{GHz}, c=3 \times 10^{8} \mathrm{~m} / \mathrm{s}$, and $0<r_{u}<100$ meters, the curve fitting parameters for $45^{\circ} \leq \theta_{U} \leq 90^{\circ}$ are $\left(a_{2}, b_{2}, c_{2}\right)=\left(7180,2,7.182 \times 10^{7}\right)$ in suburban environment, $\left(a_{2}, b_{2}, c_{2}\right)=\left(8584,2,8.838 \times 10^{7}\right)$ in urban environment, and $\left(a_{2}, b_{2}, c_{2}\right)=\left(4.73,4.033,1.125 \times 10^{8}\right)$ in dense urban environment, respectively. In the simulation, the RWP model is adopted by directly generating the random distances in (6) and (7), and not the random waypoints. Then, they were used 


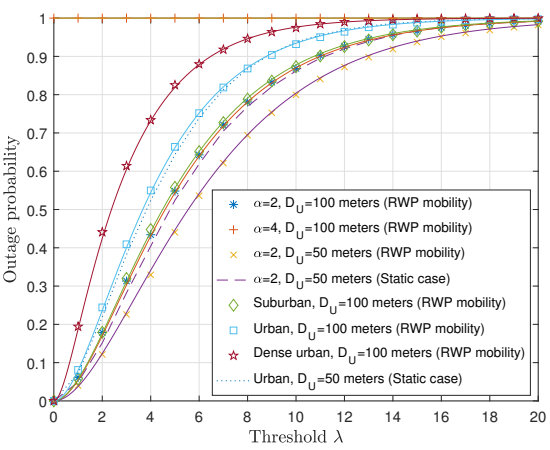

Fig. 2. The outage probability versus threshold $(\lambda)$ for $\alpha=\{2,4\}$ and different propagation environments for $m=2$ in noise-only scenario.

in (1)-(3) for the simulation of the received power. The solid lines represent the analytical results using (9)-(11) and (13)(16) while the markers represent the simulation results.

In Fig. 2, we depict the impact of path-loss exponent and urban propagation environments on the outage performance where we assume a mobility scenario with a maximum distance of $D_{U}=100$ meters, compared with a static system where the ground user is deployed at half of the maximum distance. In the RWP model, the waypoints are uniformly chosen around the center but its probability density decreases towards the boundary of the circle [4, Fig.5]. Consequently, the mobile user most likely locates near the mean distance $\bar{r}$ which can be approximated as $\bar{r}=\frac{D_{U}}{2}$ in the steady-state. This property is confirmed by our results, as shown in Fig. 2. The outage performances of a mobile user randomly moving within $D_{U}=100$ meters are approximately the same as those of a static user fixed at 50 meters. In addition, we observe better outage performance for $\alpha=2$ because the received power in (1) is higher as compared with $\alpha=4$. Also, for the probabilistic LOS model, the outage performance deteriorates as the power loss increases accordingly with dense urban, urban and suburban environments.

In Fig. 3, we examine the effect of Nakagami- $m$ fading parameters and the amount of interference generated by both the stationary and the mobile interfering users on the outage performance. For the same $D_{I}$, the outage performance improves with the static interferers because the separation distance between desired and interfering user increases. Also, as $m$ increases, the fading severity decreases for the interfering user links and in turn, the outage probability increases due to the dominance of the interference in the system.

\section{CONCLUSION}

In this letter we have derived the closed-form expressions for the CDF and analyzed the outage performance for the uplink of UAV communication system by using the RWP mobility model and the Nakagami- $m$ channel fading model. We have extended the outage analysis to the interferencelimited scenario by using the Gamma approximation. Thus, this letter provides useful design guidelines to quantify the effect of the user mobility, propagation environment, channel fading, and co-channel interference on the communication

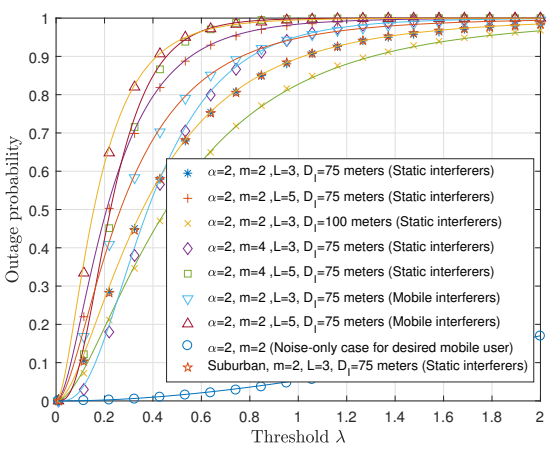

Fig. 3. The outage probability versus threshold $(\lambda)$ for $D_{U}=100$ meters, $m=\{2,4\}$ and $L=\{3,5\}$ with static and mobile interferers.

performance. Our study considers a single UAV at the cell center and ignores factors, such as UAV mobility and drift due to air turbulence. They can change the GA propagation distance and thus, affect the communication performance. However, they are beyond the scope of this letter item. Future extensions include the UAV placement optimization in the presence of the user mobility.

\section{REFERENCES}

[1] K. Govindan, K. Zeng, and P. Mohapatra, "Probability density of the received power in mobile networks," IEEE Trans. Wireless Commun., vol. 10, no. 11, pp. 3613-3619, Nov. 2011.

[2] V. A. Aalo, C. Mukasa, and G. P. Efthymoglou, "Effect of mobility on the outage and BER performances of digital transmissions over Nakagami-m fading channels," IEEE Trans. Veh. Technol., vol. 65, no. 4, pp. 2715-2721, Apr. 2016.

[3] T. Camp, J. Boleng, and V. Davies, "A survey of mobility models for ad hoc network research," Wireless Commun. and mobile comput., vol. 2, no. 5, pp. 483-502, Aug. 2002.

[4] E. Hyytiä, P. Lassila, and J. Virtamo, "Spatial node distribution of the random waypoint mobility model with applications," IEEE Trans. Mobile Comput., vol. 5, no. 6, pp. 680-694, Jun. 2006.

[5] A. Gupta and P. Garg, "Statistics of SNR for an indoor VLC system and its applications in system performance," IEEE Commun. Lett., vol. 22, no. 9, pp. 1898-1901, Sept. 2018.

[6] J. Tang, M. Dabaghchian, K. Zeng, and H. Wen, "Impact of mobility on physical layer security over wireless fading channels," IEEE Trans. Wireless Commun., vol. 17, no. 12, pp. 7849-7864, Dec. 2018.

[7] N. Cao, Y. Chen, and Z. Yang, "Secrecy outage probability with randomly moving interferers in Nakagami- $m$ fading," IEEE Commun. Lett., vol. 23, no. 1, pp. 76-79, Jan. 2019.

[8] P. K. Sharma and D. I. Kim, "Coverage probability of 3D mobile UAV networks," IEEE Wireless Commun. Lett., vol. 8, no. 1, pp. 97-100, Feb. 2019.

[9] A. Al-Hourani, S. Kandeepan and S. Lardner, "Optimal LAP altitude for maximum coverage," IEEE Wireless Commun. Lett., vol. 3, no. 6, pp. 569-572, Dec. 2014.

[10] H. Sallouha, M. M. Azari, and S. Pollin, "Energy-constrained UAV trajectory design for ground node localization," in Proc. IEEE GLOBECOM, Abu Dhabi, UAE, Jun. 2018, pp. 1-7.

[11] A. A. Khuwaja, Y. Chen, N. Zhao, M.-S. Alouini, and P. Dobbins, "A survey of channel modeling for UAV communications," IEEE Commun. Surv. Tuts., vol. 20, no. 4, pp. 2804-2821, 4th Quart. 2018.

[12] T. S. Rappaport, Wireless Communications: Principles and Practice, Prentice Hall, 2001.

[13] Y. Zeng, R. Zhang, and T. J. Lim, "Wireless communications with unmanned aerial vehicles: Opportunities and challenges," IEEE Commun. Mag., vol. 54, no. 5, pp. 36-42, May 2016.

[14] I. S. Gradshteyn and I. M. Ryzhik, Table of Integrals, Series, and Products, 6th Ed. San Diego, CA: Academic Press. 2000.

[15] V. V. Chetlur and H. S. Dhillon, "Downlink coverage analysis for a finite 3-D wireless network of unmanned aerial vehicles," IEEE Trans. Commun., vol. 65, no. 10, pp. 4543-4558, Oct. 2017. 